\title{
2-Arachidonoylglycerol Enrichment Reduced Epileptiform Activity of the Rat Hippocampus Induced with Pentylenetetrazol
}

\author{
Parisa Zareie $^{1 \text { (D) }, \text { Mehdi Sadegh }}{ }^{2 *(D)}$, Homeira Moradi-Chameh ${ }^{2}$ (D) \\ 1. Dept. of Physiology, Faculty of Medicine, Arak University of Medical Sciences, Arak, Iran \\ 2. Dept. of Fundamental Neurobiology, Krembil Research Institute, Toronto, Ontario, Canada
}

\begin{tabular}{l}
\hline Article Info \\
\hline doi 10.30699/jambs.26.118.21 \\
Received: 2017/10/22; \\
Accepted: 2018/08/18; \\
Published Online: 01 Sep 2018; \\
Use your device to scan and read the \\
article online \\
\end{tabular}

Corresponding Information: Mehdi Sadegh,

Dept. of Physiology, Faculty of Medicine, Arak University of Medical Sciences, Arak, Iran

E-mail: m.sadegh@arakmu.ac.ir

\begin{abstract}
Background \& Objective: 2-arachidonoylglycerol (2-AG) and anandamide (AEA) are two major endocannabinoids. Using inhibitors of the enzymatic pathways involved in the elimination of 2-AG and AEA as well as synthetic 2-AG, we examined the effectiveness of these endocannabinoids on epileptiform activity induced in Wistar rats by pentylenetetrazol (PTZ).
\end{abstract}

Materials \& Methods: Adult male Wistar rats were used in this study. Epileptiform activity was induced in adult male Wistar rats by PTZ injection $(20 \mathrm{mg} / \mathrm{kg}$, i.p.). To inhibit 2-AG degradation WWL70 and JJKK048 (JJKK048: $1 \mathrm{mg} / \mathrm{kg}$, WWL70: 5 $\mathrm{mg} / \mathrm{kg}$, i.p.) were used. To inhibit AEA elimination, URB597 and LY2183240 (URB597: $1 \mathrm{mg} / \mathrm{kg}$, LY2183240: $2.5 \mathrm{mg} / \mathrm{kg}$, i.p.) were used. Synthetic 2-AG was also examined (1 mg/kg, i.p.) before the PTZ injection. All drugs were dissolved in DMSO as vehicle and injected (i.p.) 15 minutes before the PTZ injection. Latency to onset and duration of the epileptiform activity were considered for statistical analysis.

Results: Injection of (JJKK048+WWL70) before the PTZ significantly increased latency to onset of the epileptiform activity $(P<0.01)$, while reduced duration of the epileptiform activity in comparison to the vehicle $(P<0.05)$. In addition, 2-AG administration significantly increased latency to onset of the epileptiform activity $(P<0.05)$ and reduced duration of the epileptiform activity in comparison to the vehicle $(P<0.01)$. However, these indexes did not show significant changes when URB597+LY2183240 were injected before the PTZ $(P>0.05)$.

Conclusion: It seems increased level of 2-AG but not AEA, effectively decreases PTZ induced epileptiform activity of the hippocampus.

Keywords: Glyceryl 2-arachidonate Anandamide, Endocannabinoids, Pentylenetetrazole, Epilepsy

\section{Introduction}

Epilepsy is among the most common neurological disorders which occurs in more than $1 \%$ of individuals around the world (1). The current therapeutic methods are not effective at least in $30 \%$ of patients and still there are cases with uncontrolled seizures even after the administration of antiepileptic drugs (2). Thus, novel therapeutic strategies are required to control previously pharmacoresistant epilepsies.

Natural cannabis has a long history in medicine (3). Cannabinoid derivatives were used for a variety of disorders such as inflammation, pain treatment, and mood disorders (3). Recently, non-psychoactive cannabinoids were proposed to be anti-convulsive agents $(4,5)$.

The endogenous cannabinoids exist in the nervous system and are known collectively as the endocannabinoids (eCB) system which includes at least two endogenous ligands, 2-arachidonoylglycerol (2-AG) and
$\mathrm{N}$-arachidonoylethanolamine (anandamide; AEA) (6). This eCB system has a significant neuromodulatory role in the neural system and participates in many physiological functions to adjust them in normal and pathological situations (7). In nervous system $2-A G$ is produced by diacylglycerol lipase (DAGL) from diacylglycerol (DAG). In addition, monoacylglycerol lipase (MAGL) mainly hydrolyzes and eliminates 2AG. Recently, a serine hydrolase a/b-hydrolase domain ABHD6 was recognized to be involved in hydrolyzing and inactivating $2-A G(8)$. AEA is primarily created by $\mathrm{N}$-arachidonyl-phosphatidy-lethanolamine phospholipase D (NAPE-PLD) from N-arachidonylphophatidyl-ethanolamine (NAPE) and is hydrolyzed and degraded through fatty acid amide hydrolase (FAAH) (9). Therefore, both FAAH and MAGL inhibitors are able to exaggerate temporal and spatial activity of the endogenous $\mathrm{eCB}$ and could be considered as therapeutic agents. 
More recent studies endorsed anti-epileptic and antiseizure effects of the eCB system in rodents $(10,11)$. Recently we had shown anticonvulsive effect of $2-A G$ in a model of tonic- clonic seizure $(12,13)$. In addition, it was reported that ABHD6 blockade, which was supposed to increase 2-AG levels, reduced behavioral and electrophysiological seizure parameters in the spontaneous genetic model and PTZ model of seizure (14). However, as ABHD6 is not the only enzyme responsible for the 2-AG degradation, in the current experiment we designed to block both presynaptic and postsynaptic enzymes which are responsible for the inactivation of 2-AG by simultaneous antagonizing MAGL and ABHD6. In addition, we designed to investigate AEA as another endocannabinoid ligand. Therefore, we blocked AEA elimination using FAAH inhibitor and AEA transporter blocker. We also applied synthetic 2-AG to enhance its level exogenously. We considered to examine all these treatments on the epileptiform activity of the hippocampus CA1 induced by PTZ.

\section{Materials and Methods}

\section{Animals}

In this experimental study we used adult male Wistar rats (170-200 g). Rats were kept in 12-h light/dark cycles with free access to food and water. Experimental procedures with animal use were done according to the Guide for the Care and Use of Laboratory Animals (8th edition; National Academies Press; 2011) and approved by the Review Board and Ethics Committee of Arak University of Medical Sciences (IR.ARAKMU. REC.1394.225). We decided to reduce the number of animals and their pain.

\section{Chemicals}

To prevent enzymatic degradation of 2-AG, WWL70 (a potent inhibitor of ABHD6; Tocris, USA) and JJKK048 (a potent and selective MAGL inhibitor; Tocris, USA) were used. To stop anandamide elimination, URB597 (a potent and selective FAAH inhibitor; Santa Cruz Biotechnology, USA) and LY2183240 (a potent and competitive inhibitor of anandamide reuptake; Cayman chemical, USA) were used. To examine exogenous $2-A G$ on seizure parameters, 2-Arachidonoylglycerol (2-AG; Tocris, USA) was used.

All chemicals were dissolved in DMSO as vehicle (Sigma-Aldrich, USA) and were injected (i.p.) 15 minutes before the PTZ injection. All animals received the same volume of DMSO $(0.8 \mathrm{ml} / \mathrm{rat})$. Based on previous studies, JJKK048: $1 \mathrm{mg} / \mathrm{kg}$ (15), WWL70: 5 mg/kg (16), URB597: 2.5 mg/kg (17), LY2183240: 2.5 $\mathrm{mg} / \mathrm{kg}$ (18), 2-AG: $1 \mathrm{mg} / \mathrm{kg}$ (19) were used. In the sham experimental group, PTZ was injected after DMSO.

Experimental Groups were as below:

(1) PTZ (sham); n=7

(2) DMSO+PTZ (vehicle); $\mathrm{n}=7$
(3) URB597+ LY2183240+DMSO+PTZ (blocking the AEA elimination); $n=7$

(4) WWL70+JJKK048+DMSO+PTZ (blocking the 2AG degradation); $\mathrm{n}=7$

(5) 2-AG+DMSO+PTZ (synthetic 2-AG); $\mathrm{n}=7$

All chemicals were injected (i.p.) 15 minutes before the PTZ injection.

\section{PTZ Induced Epileptiform EEG Activity}

Under ketamine/xylazine $(60$ and $10 \mathrm{mg} / \mathrm{kg}$ respectively; i.p.) anesthesia, 35 adult male Wistar rats were stereotaxically implanted with a unipolar recording electrode into CA1 of hippocampus of the right hemisphere, (A, -3.8 $\mathrm{mm}$ from bregma; $\mathrm{L}, 2.3 \mathrm{~mm}$ from bregma; V, $2.2 \mathrm{~mm}$ from skull) according to the atlas of Paxinos and Watson. Electrodes (stainless steel, teflon coated, $127 \mu \mathrm{m}$ in diameter, A.M. systems, Inc., USA) were insulated except at their tips. Stainless steel screws were also positioned in the skull above the frontal and occipital cortices. These served as reference and ground electrodes. The entire assembly was sealed and fixed to the skull with dental acrylic.

Epileptiform EEG activity was continuously recorded 5-15 minutes before and 30 minutes after the PTZ injection $(20 \mathrm{mg} / \mathrm{kg}$; i.p), using a homemade PC based data acquisition system (sampling rate was $1 \mathrm{kHz}$ ). Signals were amplified $(\times 1000$ times; high and low pass filtered at 1 and $200 \mathrm{~Hz}$, respectively) and analyzed offline with a homemade software.

\section{Statistical Analysis}

Data were analyzed by GraphPad Prism software. First normal distribution was tested using the D'AgostinoPearson normality test. To compare more than two groups one-way ANOVA and Bonferroni post-test were applied and $\mathrm{P}$-value $<0.05$ were consider as significant confidence. Data are presented as a mean \pm S.E.M.

\section{Results}

2-AG Degradation Blockade or Synthetic 2AG Administration Decreased Duration of Epileptiform Activity of Hippocampus CA1.

Intraperitoneal injection of a sub-convulsive dose of PTZ caused epileptiform activity during the EEG recording from the CA1 of the hippocampus (Figure 1). When DMSO as the vehicle was injected (i.p.) alone, 15 minutes prior to the PTZ injection, no significant differences were appeared in the latency to onset (Figure 2) and duration (Figure 3) of the epileptiform activity in comparison with the control group. Injections of URB597+LY2183240 (to block the AEA elimination) also revealed no significant alterations on latency to onset (Figure 2) and duration (Figure 3) of the epileptiform activity when compared with the vehicle group. However, blocking the 2-AG degradation (using the JJKK048+WWL70) caused a significant increase in the latency to onset (Figure 2; $P<0.01$ ), while reduced the duration (Figure $3 ; P<0.05$ ) 
of the epileptiform activity in comparison with the vehicle group. Moreover, synthetic 2-AG administration revealed an analogous effect and significantly increased the latency to onset (Figure 2; $P<0.05$ ), while reduced the duration (Figure $3 ; P<0.01$ ) of the epileptiform activity in comparison with the vehicle group. The analyzed data using the one-way ANOVA with Bonfferoni post-test is presented as Mean+SEM. All groups were considered with an $n=7$.

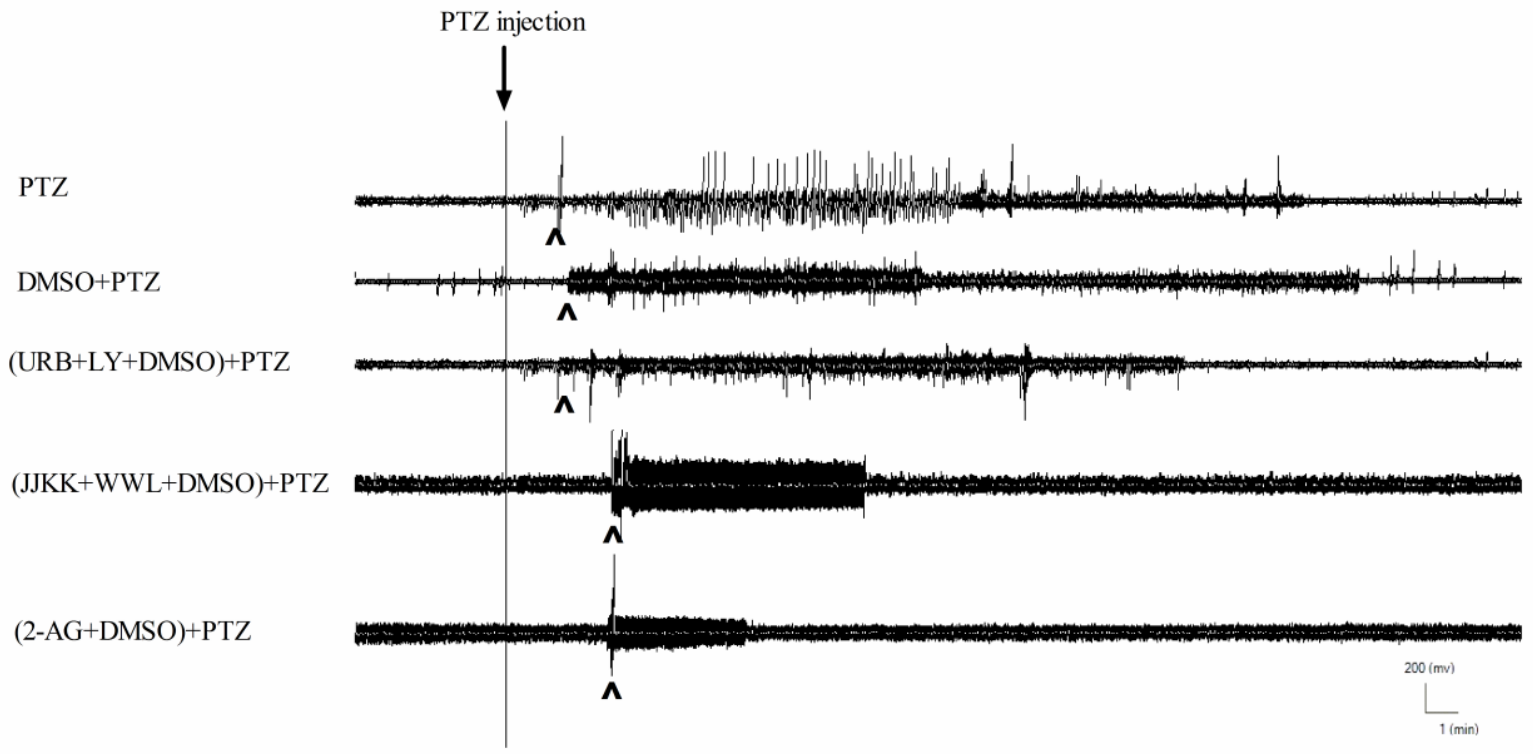

Figure 1. Sample traces selected from the recorded data of experimental groups for demonstration. PTZ injection $(20 \mathrm{mg} / \mathrm{kg}$; i.p.) pointed with the vertical bar and the onset of the epileptiform activity illustrated with $(\wedge)$. As demonstrated in these sample traces the onset point and the duration of the epileptiform activity show differences between experimental groups.

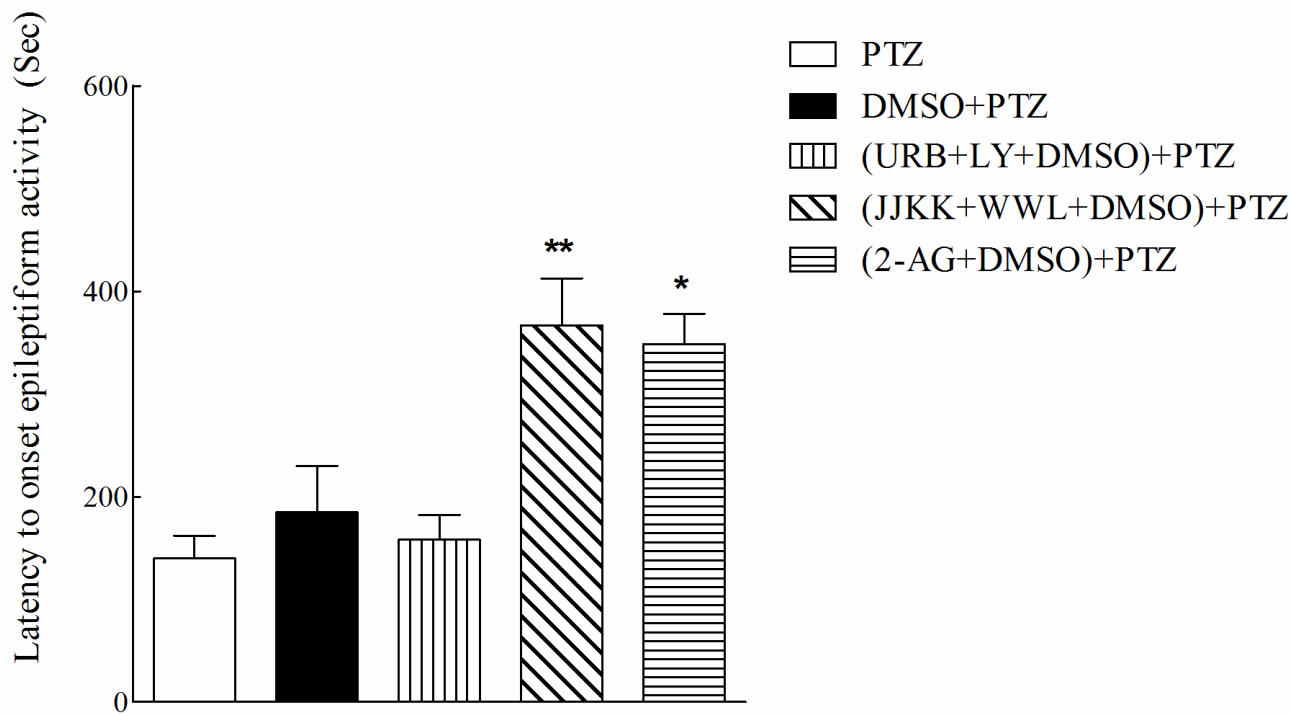

Figure 2. Delay to onset of the epileptiform activity of hippocampus CA1 decreased with intraperitoneal injection of 2-AG or blockade the 2-AG degradation. Latency to onset of the epileptiform activity after PTZ injection did show significant differences. As demonstrated blocking the 2-AG degradation with using the JJKK048+WWL70 or administrating the synthetic 2AG significantly increased the delay to onset of the epileptiform activity when compared with vehicle group. $n=7$ in groups; $* * P<0.01$ and $* P<0.05$ by one-way ANOVA with bonferroni's post-hoc test; error bars mean \pm SEM. 


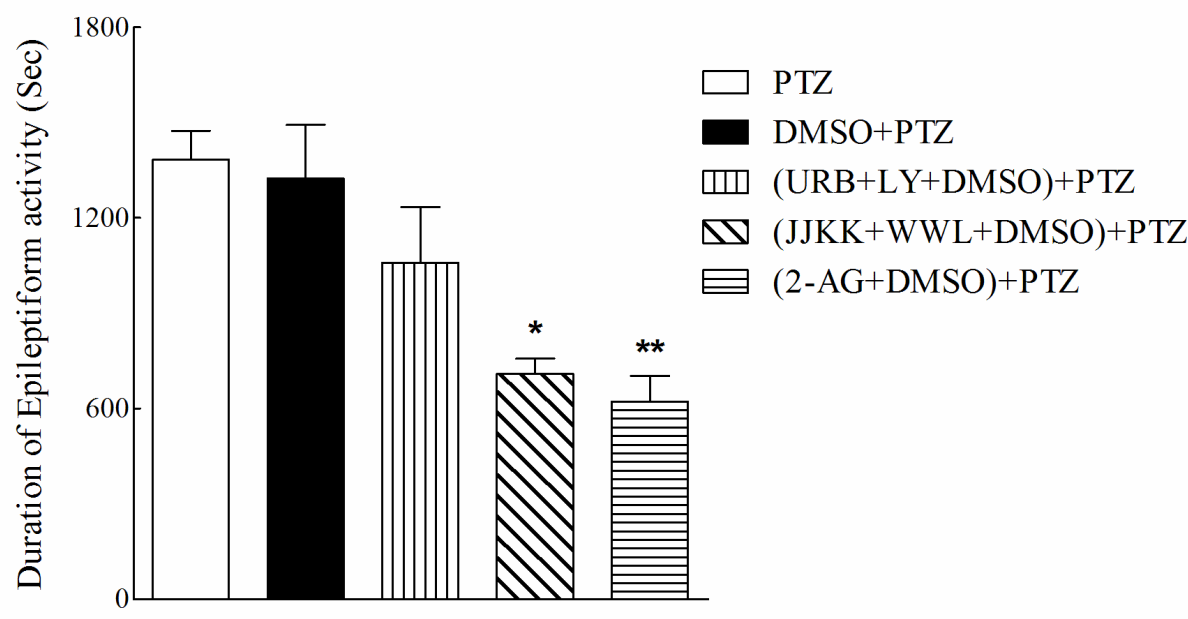

Figure 3. Duration of the epileptiform activity of hippocampus CA1 increased with intraperitoneal injection of 2-AG or blockade the 2-AG degradation. Quantification of epileptiform activity revealed a significant decrease in duration of epileptiform activity in experimental groups with synthetic 2-AG administration or blockade the 2-AG degradation (using the JJKK048+WWL70), when compared with vehicle group. $\mathrm{n}=7$ in groups; $* * P<0.01$ and $* P<0.05$ by one-way ANOVA with bonferroni's post-hoc test; error bars mean \pm SEM.

\section{Discussion}

We examined the consequences of increased levels of 2-AG and AEA, on a PTZ model of epileptic activity. Our results revealed the effectiveness of enhanced 2-AG but not AEA on epileptiform activity induced by PTZ. According to the results, blocking the 2-AG degradation or even administrating synthetic $2-\mathrm{AG}$ had advantageous effects on epileptiform activity of the hippocampus. Both latency to onset and duration of these activity were significantly decreased. However, preventing the AEA abolition in this study had no significant effect on seizure indexes. Our findings proved evidence for antiepileptic effects of both endogenous and exogenous 2-AG.

In association with our previous investigations $(12,13)$, herein we applied a subthreshold dose of PTZ to induce epileptiform activity in the hippocampal formation with no obvious behavioral seizure activity. This had been introduced as a model for absence epilepsy in human (20, 21). While convulsive dose of PTZ provokes seizure activates with apparent tonic-clonic contraction in the body. In addition, local EEG recording from the hippocampus show the excitability of this structure, therefore, the result of the current experiment would be benefited in the line of absence seizure and neuronal hyperexcitability disorders.

MAGL, ABHD6 are well recognized members of the endocannabinoids signaling system and responsible for controlling the 2-AG levels (8). Our results show that 2AG degradation blockade by JJKK048+WWL70 exerts an antiepileptic effect as reduced the epileptiform activity of the hippocampus, which was in agreement with the results from the study by Naydenov et al. (14), as they were reported that blocking the ABHD6 by WWL70 produced antiepileptic effect (14). However, considering the importance of MAGL in the 2-AG degradation pathway (8), we used blockers for both MAGL (blocked by JJKK048) and ABHD (blocked by WWL70).
Although both these enzymes regulate 2-AG levels, but they are located at different synaptic signaling pathways. ABHD6 is a postsynaptic enzyme and present in the AMPA channel macromolecular complex (22). In accordance, selective ABHD6 inhibition has been shown to be effective in the PTZ model. While, MAGL is a presynaptic enzyme and colocalize with $\mathrm{CB} 1$ receptors on axon terminals (23). Although, ABHD6 and MAGL play different synaptic roles the combined administration of their inhibitors in our study may lead to a novel understanding of how 2-AG signaling regulates epileptic network activity. Antiepileptic effects of ABHD6 blockade have been reported to be independent of $\mathrm{CB}$ receptors (14).

Many of the previous investigations which were shown antiepileptic properties for non-psychoactive phytocannabinoids, have also reported that these therapeutic effects of phytocannabinoids, to be independent of the $\mathrm{CB}$ receptors (24). The involvement of some other possible pathways such as activation of the $5-\mathrm{HT} 1_{\mathrm{A}}$ or blocking the T-type $\mathrm{Ca}^{++}$channels have been discussed in a number of previous studies (3). However, other studies had shown that increasing the 2-AG levels in the CNS produced disruption of the memory performance, which occurs via a CB1R- dependent mechanism (25). According to direct and positive allosteric modulation of $\mathrm{GABA}_{\mathrm{A}}$ receptors by $2-A G$ (26) these antiepileptic and anticonvulsive effects of blocking the 2-AG degradation might occur through the modulation of $\mathrm{GABA}_{\mathrm{A}}$ receptors. However, the existence of other related signaling pathways which might be influenced by 2-AG or other unknown mechanisms are not improbable. Further investigations should be designed to precisely show if the anticonvulsive 
effect of 2-AG proceeds directly through $\mathrm{GABA}_{\mathrm{A}}$. Moreover, it should be taken into considered if there is a synergic effect between $\mathrm{GABA}_{\mathrm{A}}$ agonist and 2-AG.

To confirm the antiepileptic effect of 2-AG, we applied exogenous 2-AG before the PTZ injection and like the first report, our results verified antiepileptic effects of 2AG. We had previously published data about the direct application of 2-AG against acute seizures induced by PTZ (12). In addition, there are a number of studies which have demonstrated the exogenous application of $\mathrm{N}$ palmitoylethanolamide (as endocannabinoids candidate) and its anticonvulsive and antiepileptic effects (27). Although, the inhibition of the refractory status epilepticus using anandamide has been previously observed in the hippocampal neuronal culture model of epilepsy (28), the results from our experiments demonstrate the blockade the AEA elimination has no anticonvulsive effect. However, as we have only used a single dose of the FAAH inhibitor the lack of an effect does not necessarily mean that anandamide signaling is not involved in the regulation of network activity during increased excitability states. FAAH knockouts exhibit pronounced epileptic seizure susceptibility (29) and anandamide were previously found to be a fully efficacious anticonvulsant and dependent on CB1 in the maximal electroshock model (29).

Controversial results from a recent study (30) showed that inhibition of MAGL (enzyme primarily responsible for degrading 2-AG) by JZL184 caused increase in spontaneous recurrent seizures, hippocampal damage and cognitive impairment. In contrast, treatment with RHC80267 (an inhibitor of diacylglycerol lipase, which mainly produced 2-AG) had beneficial effects on spontaneous recurrent seizures, cognitive performances, and seizure-induced hippocampal neuronal loss in mice models with chronic seizures (31). Considering the experimental protocol which was used in their study, these contradictory results might be explained. An animal model of status epilepticus which was induced by pilocarpine was used in their study (32). According to literature, the mechanism of pilocarpine induced seizures and epilepsy is completely different from PTZ (33). As a $\mathrm{GABA}_{\mathrm{A}}$ antagonist, PTZ provokes seizure activity through modifying the inhibitory circuits in the CNS, while pilocarpine acts as a muscarine receptor agonist and works through modifying the cholinergic system of the CNS (34). As was discussed above, 2-AG anticonvulsive effects occur via $\mathrm{GABA}_{\mathrm{A}}$ receptors. Therefore, it might be anticipated that blocking the 2-AG degradation has beneficial effects in the models with $\mathrm{GABA}_{\mathrm{A}}$ abnormality but less likely in other situations such as pilocarpine induced epilepsy. The study also involved applying the blockers chronically for 1 week. It has been shown that prolonged exposure with cannabinoids results in the development of tolerance to the anticonvulsant effects of cannabinoids and an exacerbation of seizure activity in a hippocampal neuronal culture model (35). That being said, the prolonged increase in the 2-AG levels may possibly change or even convert the results. A study on the kainic acid model of epilepsy has been shown to have activity-dependence of 2-AG synthesis (11). Due to weak and local enzymatic activity of both MAGH and ABHD, spatiotemporal specificity of these blockades exhibits a favorable safety profile and maybe amenable to long-term use for the treatment of seizures.

As a limitation of our study, we did not use behavioral tests to examine emotional or cognitive effects of these administered chemicals. However, because eCB system plays a role in cognitive and emotional activities of the CNS (7), it should be considered in the future studies.

\section{Conclusion}

2-AG which is assumed to be the main ligand of the eCB system has anticonvulsive effects when increased endogenously or exogenously and might be considered as a potential therapeutic target for epilepsy. On the contrary, anandamide did not show such effects in this study.

\section{Acknowledgment}

This research experiment was supported by Arak University of Medical Sciences (funding number: 1075). There are no conflicts of interests for the authors.

\section{Conflict of Interest}

Authors declare no conflict of interests.

\section{References}

1. De Boer HM, Mula M, Sander JW. The global burden and stigma of epilepsy. Epilepsy Behav. 2008; 12(4): 540-6. [DOI:10.1016/j.yebeh.2007.12.019] [PMID]

2. Hitiris N, Mohanraj R, Norrie J, Sills GJ, Brodie MJ. Predictors of pharmacoresistant epilepsy. Epilepsy Res. 2007; 75: 192-6. [DOI:10.1016/j.eplepsyres.2007.06.003] [PMID]

3. Hill AJ, Williams CM, Whalley BJ, Stephens GJ. Phytocannabinoids as novel therapeutic agents in CNS disorders. Pharmacol Ther. 2012; 133(1): 79-97. [DOI:10.1016/j.pharmthera.2011.09.002] [PMID]

4. Russo EB. Cannabis and epilepsy: An ancient treatment returns to the fore. Epilepsy Behav. 2017; 70: 292-7. [DOI:10.1016/j.yebeh.2016.09.040] [PMID]

5. dos Santos RG, Hallak JE, Leite JP, Zuardi AW, Crippa JA. Phytocannabinoids and epilepsy. J Clin Pharm Ther. 2014; 40: 135-43. [DOI:10.1111/jcpt.12235] [PMID]

6. Ligresti A, Cascio MG, Di Marzo V. Endocannabinoid metabolic pathways and enzymes. Curr Drug Targets CNS Neurol Disord. 2005; 4(6): 615-23. [DOI:10.2174/156800705774933104] [PMID]

7. Battista N, Di Tommaso M, Bari M, Maccarrone M. The endocannabinoid system: an overview. Front Behav Neurosci. 2012; 6: 9. [DOI:10.3389/fnbeh.2012.00009] [PMID] [PMCID]

8. Savinainen J, Saario S, Laitinen J. The serine hydrolases MAGL, ABHD6 and ABHD12 as guardians of 2arachidonoylglycerol signalling through cannabinoid receptors. Acta physiologica. 2012; 204(2): 267-76. 
[DOI:10.1111/j.1748-1716.2011.02280.x] [ [PMCID]

9. Basavarajappa BS. Critical enzymes involved in endocannabinoid metabolism. Protein Pept Lett. 2007; 14(3): 237-46. [DOI:10.2174/092986607780090829]

10. Sugaya Y, Yamazaki M, Uchigashima M, et al. Crucial Roles of the Endocannabinoid 2-Arachidonoylglycerol in the Suppression of Epileptic Seizures. Cell Rep. 2016; 16(5): 1405-15. [DOI:10.1016/j.celrep.2016.06.083] [PMID]

11. Fezza F, Marrone MC, Avvisati R, et al. Distinct modulation of the endocannabinoid system upon kainic acid-induced in vivo seizures and in vitro epileptiform bursting. Mol Cell Neurosci. 2014; 62: 1-9. [DOI:10.1016/j.men.2014.07.003] [PMID]

12. Zareie P, Sadegh M, Palizvan M. The effect of 2-archidonyl glycerol (2-AG) as an endocannabinoid on tonic- clonic seizures induced by pentylenetetrazol (PTZ). Zanjan Univ Med Sci J. 2017; 25(109): 11-22.

13. Zareie $P$, Sadegh M, Palizvan M. Investigating the effect of enzymatic elimination of endocannabinoids inhibitors on tonic- colonic seizure provoked by PTZ. Babol Univ Med Sci. 2016; 18(12): 49-56.

14. Naydenov AV, Horne EA, Cheah CS, et al. ABHD6 blockade exerts antiepileptic activity in PTZ-induced seizures and in spontaneous seizures in R6/2 mice. Neuron. 2014; 83(2): 361-71. [DOI:10.1016/j.neuron.2014.06.030] [PMID] [PMCID]

15. Aaltonen N, Savinainen JR, Ribas CR, et al. Piperazine and piperidine triazole ureas as ultrapotent and highly selective inhibitors of monoacylglycerol lipase. Chem Biol. 2013; 20(3): 379-90. [DOI:10.1016/j.chembiol.2013.01.012] [PMID]

16. Wen J, Ribeiro R, Tanaka M, Zhang Y. Activation of CB2 receptor is required for the therapeutic effect of ABHD6 inhibition in experimental autoimmune encephalomyelitis. Neuropharmacol. 2015; 99: 196-209. [DOI:10.1016/j .neuropharm.2015.07.010] [PMID]

17. Hasanein P, Ghafari-Vahed M. Fatty acid amide hydrolase inhibitor URB597 prevented tolerance and cognitive deficits induced by chronic morphine administration in rats. Behav Pharmacol. 2016;27(1): 37-43. [DOI:10.1097/FBP.0000000000000179] [PMID]

18. Maione S, Morera E, Marabese I, et al. Antinociceptive effects of tetrazole inhibitors of endocannabinoid inactivation: cannabinoid and non-cannabinoid receptormediated mechanisms. Br J Pharmacol. 2008; 155(5): 77582. [DOI:10.1038/bjp.2008.308] [PMID] [PMCID]

19. Wakley AA, Rasmussen EB. Effects of cannabinoid drugs on the reinforcing properties of food in gestationally undernourished rats. Pharmacol Biochem Behav. 2009; 94(1): 30-6. [DOI:10.1016/j.pbb.2009.07.002] [PMID]

20. Depaulis A, Snead OC, Marescaux C, Vergnes M. Suppressive effects of intranigral injection of muscimol in three models of generalized non-convulsive epilepsy induced by chemical agents. Brain Res. 1989; 25; 498(1): 64-72. [DOI:10.1016/0006-8993(89)90399-5]

21. Snead OC, Banerjee PK, Burnham M, Hampson D. Modulation of absence seizures by the GABA(A) receptor: a critical rolefor metabotropic glutamate receptor 4
(mGluR4). J Neurosci. 2000; 20(16): 6218-24. [DOI:10.1523/JNEUROSCI.20-16-06218.2000]

22. Schwenk J, Harmel N, Brechet A, et al. High-resolution proteomics unravel architecture and molecular diversity of native AMPA receptor complexes. Neuron. 2012; 24; 74(4): 621-33. [DOI:10.1016/i.neuron.2012.03.034] [PMID]

23. Ludanyi A, Eross L, Czirjak S, et al. Downregulation of the CB1 cannabinoid receptor and related molecular elements of the endocannabinoid system in epileptic human hippocampus. J Neurosci. 2008; 28(12): 2976-90. [DOI:10.1523/JNEUROSCI.4465-07.2008] [PMID]

24. Hill TD, Cascio MG, Romano B, et al. Cannabidivarin-rich cannabis extracts are anticonvulsant in mouse and rat via a CB1 receptor-independent mechanism. Br J Pharmacol. 2013; 170(3): 679-92. [DOI:10.1111/bph.12321] [PMID] [PMCID]

25. Griebel G, Pichat P, Beeske S, et al. Selective blockade of the hydrolysis of the endocannabinoid 2arachidonoylglycerol impairs learning and memory performance while producing antinociceptive activity in rodents. Sci Rep. 2015; 5: 7642. [DOI:10.1038/srep07642] [PMID] [PMCID]

26. Sigel E, Baur R, Racz I, et al. The major central endocannabinoid directly acts at $\operatorname{GABA}(\mathrm{A})$ receptors. Proc Natl Acad Sci U S A. 2011;108(44): 18150-5. [DOI:10.1073/pnas.1113444108] [PMID] [PMCID]

27. Citraro R, Russo E, Scicchitano F, et al. Antiepileptic action of N-palmitoylethanolamine through CB1 and PPAR-alpha receptor activation in a genetic model of absence epilepsy. Neuropharmacol. 2013; 69: 115-26. [DOI:10.1016/j.neuropharm.2012.11.017] [PMID]

28. Deshpande LS, Blair RE, Ziobro JM, Sombati S, Martin BR, DeLorenzo RJ. Endocannabinoids block status epilepticus in cultured hippocampal neurons. Europ J Pharmacol. 2007; 558: 52-9. [DOI:10.1016/j.ejphar.2006.11.030] [PMID] [PMCID]

29. Clement AB, Hawkins EG, Lichtman AH, Cravatt BF. Increased seizure susceptibility and proconvulsant activity of anandamide in mice lacking fatty acid amide hydrolase. $\mathrm{J}$ Neurosci. 2003; 23(9): 3916-23. [DOI:10.1523/JNEUROSCI.23-09-03916.2003]

30. Corvino V, Marchese E, Podda MV, et al. The neurogenic effects of exogenous neuropeptide Y: early molecular events and long-lasting effects in the hippocampus of trimethyltintreated rats. PloS one. 2014; 9(2): e88294. [DOI:10.1371/journal.pone.0088294] [PMID] [PMCID]

31. Hoover HS, Blankman JL, Niessen S, Cravatt BF. Selectivity of inhibitors of endocannabinoid biosynthesis evaluated by activity-based protein profiling. Bioorg Med Chem Lett. 2008; 18(22): 5838-41. [DOI:10.1016/j.bmcl.2008.06.091] [PMID] [PMCID]

32. Ma L, Wang L, Yang F, et al. Disease-modifying effects of RHC80267 and JZL184 in a pilocarpine mouse model of temporal lobe epilepsy. CNS Neuro Sci Ther. 2014; 20(10): 905-15. [DOI:10.1111/cns.12302] [PMID] [PMCID]

33. Curia G, Longo D, Biagini G, Jones RS, Avoli M. The pilocarpine model of temporal lobe epilepsy. J Neuro Sci Methods. 2008; 30; 172(2-4): 143-57. [DOI:10.1016/j.jneumeth.2008.04.019] [PMID] [PMCID] 
34. Löscher W. Critical review of current animal models of seizures and epilepsy used in the discovery and development of new antiepileptic drugs. Seizure. 2011; 20(5): 359-68. [DOI:10.1016/j.seizure.2011.01.003] [PMID]

35. Blair RE, Deshpande LS, Sombati S, Elphick MR, Martin BR, DeLorenzo RJ. Prolonged exposure to WIN55,212-2 causes downregulation of the $\mathrm{CB} 1$ receptor and the development of tolerance to its anticonvulsant effects in the hippocampal neuronal culture model of acquired epilepsy. Neuropharmacology. 2009; 57(3): 208-18. [DOI:10.1016/j.neuropharm.2009.06.007] [PMID] [PMCID]

\section{How to Cite This Article:}

Zareie P, Sadegh M, Moradi-Chameh H. 2-Arachidonoylglycerol enrichment Reduced Epileptiform Activity of the Rat Hippocampus induced with Pentylenetetrazol. J Adv Med Biomed Res. 2018; 26 (118) :21-27

\section{Download citation:}

$\underline{\text { BibTeX }}|\underline{\text { RIS }}| \underline{\text { EndNote }}|\underline{\text { Medlars }}| \underline{\text { ProCite }}|\underline{\text { Reference Manager }}| \underline{\text { RefWorks }}$

\section{Send citation to:}

Mendeley 2 Zotero (i) RefWorks $\underline{\text { RefWorks }}$ 